\title{
NMR for Mixture Analysis: Concentration Ordered Spectroscopy
}

\author{
Bin Yuan, ${ }^{a}$ Zhiming Zhou, ${ }^{a}$ Bin Jiang, ${ }^{\text {a,b }}$ Ghulam Mustafa Kamal, ${ }^{c}$ Xu Zhang, ${ }^{\text {a,b,d }}$ Conggang Li, ${ }^{\text {a,b,d }}$ Xin \\ Zhou, ${ }^{\text {a,b,d }}$ Maili Liu, ${ }^{* a, b, d}$ \\ a. State Key Laboratory of Magnetic Resonance and Atomic and Molecular Physics, Wuhan Center for Magnetic Resonance, \\ Wuhan Institute of Physics and Mathematics, Innovation Academy for Precision Measurement Science and Technology, \\ Chinese Academy of Sciences, Wuhan 430071 (China) \\ b. University of Chinese Academy of Sciences, Beijing 100029, (China) \\ c. Department of Chemistry, Khwaja Fareed University of Engineering and Information Technology Rahim Yar Khan 64200 \\ (Pakistan) \\ d. Wuhan National Laboratory for Optoelectronics, Huazhong University of Science and Technology, Wuhan, 430074 \\ (China). \\ *. Corresponding Author Email: Maili Liu, ml.liu@ apm.ac.cn.
}

\begin{abstract}
A novel approach, concentration ordered NMR spectroscopy (CORDY), is being proposed based on the principle that the ratio of NMR peak area to its associated number of spins is proportional to the concentration of the assigned compound. Besides, the prior information of chemical shift distribution and lineshape characteristics of different chemical groups is ultilized to shrink the solution space. CORDY generates a pseudo 2-dimensional NMR spectrum with chemical shifts in one axis and concentrations in the other, resulting both in separation and quantitation of components in complex samples. The method was validated by applying on three samples comprising of a model mixture containing six amino acids, sugar-free Red Bull@ and human urine. It was demonstrated that CORDY could successfully provide separation up to two orders of magnitude in concentration dimension, for the samples used in current study. In addition, a combination of CORDY and DOSY (CORDY-DOSY) has been found to be more efficient in resolving the molecules with similar concentrations or self-diffusion coefficients.
\end{abstract}

Nuclear magnetic resonance (NMR) spectroscopy ${ }^{1-3}$ is considered to be a powerful tool in qualitative and quantitative analysis of mixtures, as well as studies of molecular structure, interaction and dynamics. All this information is contained in NMR peaks and can be explored by using appropriate pulse sequences that manipulate nuclear spins. In a simple case, the peak area in a fully relaxed single-pulse one dimensional (1D) NMR spectrum is proportional to the number of spins and concentration of the molecule. It is a unique feature of NMR that the resonance intensity or peak area is molecular independent, which ensures that concentrations of all components of a mixture can be determined quantitatively in one experiment. ${ }^{4-6}$

Identification of components in complex samples can be achieved by two or higher dimensional (nD) correlation experiments. ${ }^{7-9}$ The classical approaches for establishing the correlation are based on either spin-spin coupling ${ }^{10}$ or dipole-dipole coupling, ${ }^{11}$ resulting in correlation between the directly coupled functional groups or chains within a molecule, which has played a fundamental role in methodology and application of modern NMR spectroscopy. To ensure full assignment of NMR peaks of larger or complex molecules, two or more $\mathrm{nD}$ experiments are generally needed.

Diffusion ordered NMR spectroscopy (DOSY) ${ }^{12-16}$ is another type of methods widely used for mixture analysis. DOSY separates chemical shift in one dimension and molecular self-diffusion coefficient in another. As the self-diffusion coefficient is a molecular property, all peaks from one molecule will be aligned accordingly in the DOSY plot and therefore, can be distinguished from the other components. In addition, combination of diffusion and relaxation weighted NMR has been proved to be a valuable technique for improving the separation of biological mixtures. ${ }^{17-19}$ The third type of the methods, namely, statistical total correlation spectroscopy (STOCSY), ${ }^{20-23}$ was developed by Nicholson's group for identifying potential biomarkers and the molecules involved in the same pathway. STOCSY ${ }^{20}$ is based on the principle that in a set of NMR spectra, intensities of the peaks from one molecule or from the molecules that are involved in a same pathway are linearly correlated. The covariation can be measured statistically and is used to generate pseudo $2 \mathrm{D}$ plot of STOCSY.$^{20}$ In addition, electronic mobility has also been utilized in NMR (mobility spectroscopy, MOSY) to separate the charged molecules in solution. ${ }^{24-27}$ However, due to the limited dynamic ranges, the molecules with similar selfdiffusion coefficient constants, electronic mobility or concentration changes could not be fully resolved by DOSY, MOSY and STOCSY, respectively. Herein, we proposed a novel approach for constructing concentration ordered NMR spectroscopy (CORDY), for simultaneously separating and quantitating the components in a mixture.

\section{Concentration Ordered Spectroscopy (CORDY)}

For a molecule consisting of $m$ magnetically non-equivalent functional groups $\left(\mathrm{CH}, \mathrm{CH}_{2}\right.$ or $\left.\mathrm{CH}_{3}\right)$, there would be $m$ peaks in its ${ }^{1} \mathrm{H}$ NMR spectrum. Therefore, a mixture with $l$ components 
will have $m \cdot l$ peaks at maximum. The peak area, $A_{\mathrm{i}}$, is proportional to the number of magnetically equivalent protons $\left(N_{\mathrm{i}}\right)$ and molar concentration $\left(C_{\mathrm{i}}\right)$, i.e. $A_{\mathrm{i}}=k N_{\mathrm{i}} C_{\mathrm{i}}$. According to the NMR principle, the resonance index $(k)$ is non-discriminative for all peaks and molecules, and thus could be treated as unit (1.0). In this case, $C_{\mathrm{i}}$ represents relative concentration,

$$
\begin{aligned}
C_{\mathrm{i}} & =A_{\mathrm{i}} / N_{\mathrm{i}} . \\
N_{\mathrm{i}} & =\operatorname{round}\left(A_{\mathrm{i}} / C_{\mathrm{i}}, 0\right) .
\end{aligned}
$$

Where $\operatorname{round}\left(A_{\mathrm{i}} / C_{\mathrm{i}}, 0\right)$ is an operation of rounding off $A_{\mathrm{i}} / C_{\mathrm{i}}$ to the nearest integer. For ${ }^{1} \mathrm{H}$ NMR, the common value of $N_{\mathrm{i}}$ is 1 , 2 or 3 corresponding to $\mathrm{CH}, \mathrm{CH}_{2}$ or $\mathrm{CH}_{3}$. The number can be doubled $(2,4,6)$ or tripled $(3,6,9)$ in the case of magnetic equivalence. Once the proton number $\left(N_{\mathrm{i}}\right)$ is obtained, it is possible to get the concentration $\left(C_{\mathrm{i}}\right)$ associated with each peak. By plotting the chemical shift and the derived $C_{\mathrm{i}}$ as $x$ and $y$ coordinates, respectively, one gets a pseudo $2 \mathrm{D}$ spectrum of CORDY.

For constructing CORDY, the key point is the determination of $N$ for each peak. The general knowledge of $\mathrm{NMR}^{9,28}$ implies that $\mathrm{CH}_{3}$ normally appears at chemical shift range of $80.0 \sim \delta 4.0$ with the line-shape of singlet, doublet or triplet. $\mathrm{CH}$ and $\mathrm{CH}_{2}$ can be found in the range of $\delta 1.0 \sim \delta 15.0$ and $\delta 0.6 \sim \delta 5.5$, respectively, with variable line-shapes from singlet to multiplet. These essential properties are used as the first criteria. For a mixture with $m \cdot l$ peaks, it is recommended to find a peak $\left(A_{\mathrm{j}}\right)$ with known proton number $\left(N_{\mathrm{j}}\right)$ and to use the ratio as divisor:

$$
C_{\mathrm{i}} / C_{\mathrm{j}}=\left(N_{\mathrm{i}} / A_{\mathrm{i}}\right) /\left(N_{\mathrm{j}} / A_{\mathrm{j}}\right) \text {. }
$$

If peaks $\mathbf{i}$ and $\mathbf{j}$ belong to the same molecule $\left(C_{\mathrm{i}}=C_{\mathrm{j}}\right)$, one gets Eq. 3,

$$
\begin{aligned}
& N_{\mathrm{j}} A_{\mathrm{i}} / A_{\mathrm{j}}=N_{\mathrm{i}} \pm \Delta_{\mathrm{i}, \mathrm{j}}, \\
& N_{\mathrm{i}}=\operatorname{round}\left(N_{\mathrm{j}} A_{\mathrm{i}} / A_{\mathrm{j}}, 0\right) .
\end{aligned}
$$

Where $\Delta$ is the allowed error and is used as a threshold $(\leq 0.1$ or $\leq 5 \%$ ) for defining the proton number. With the obtainable peak areas and the finite number of protons associated with each peak, one can get the $N$ for each peak by using Eq. 3, as the second criteria. Therefore, the peaks with $N=1,2,3$ and $\Delta \leq$ $0.1(5 \%)$ are likely to come from the same molecule or component. A short program was written accordingly to define the proton number, to group and assign the peaks (Scheme 1).

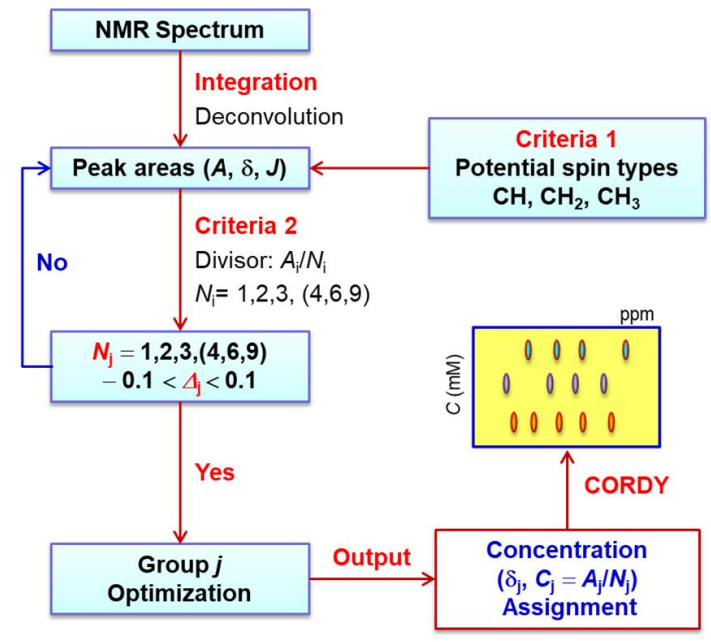

Scheme 1. Flowchart for constructing CORDY.

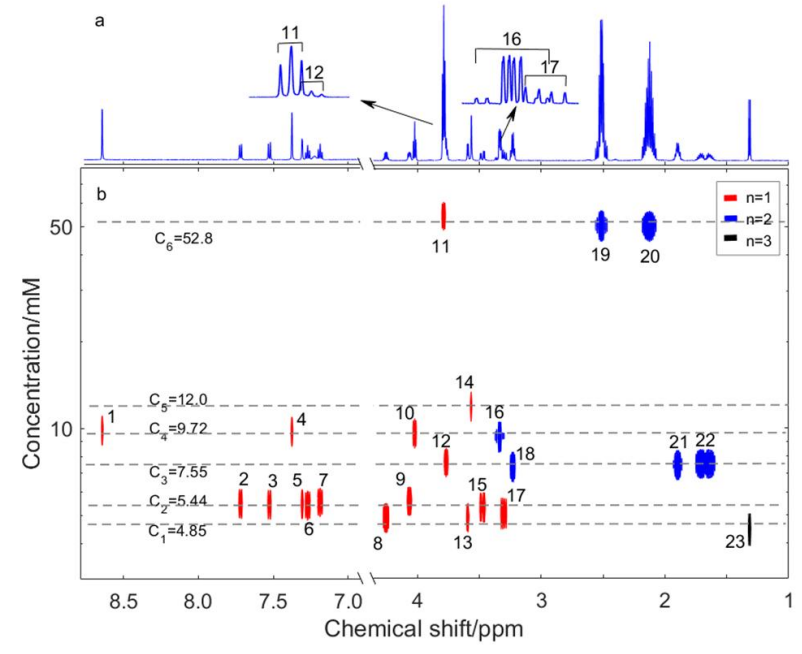

Figure 1. (a) High resolution $1 \mathrm{H}$ NMR spectrum showing the spectral region (1.0 8.7ppm) with two enlarged parts after resolution enhancement and its CORDY plot (b). The peaks are assigned as follows: L-threonine $(\mathbf{8}, \mathbf{1 3}, \mathbf{2 3})$, L-glutamate $(\mathbf{1 1}$, $19,20)$, L-histidine $(1,4,10,16)$, L-tryptophan $(2,3,5,6,7,9$, 15, 17), L-arginine $(12,18,21,22)$ and glycine $(14)$. The concentration value of components is referenced to L-threonine (4.85 mM).

At first, a model mixture consisting of six amino acids was taken as an example to optimize the process of constructing CORDY. The procedure starts with the acquisition of a fully relaxed ${ }^{1} \mathrm{H}$ NMR spectrum (Figure 1a). After careful correction of the phase and baseline, the integral values of twenty-three peaks were obtained (Table S1). Among those peaks, two overlapped regions, peaks $11 \& 12,16 \& 17$, were resolved by deconvolution. ${ }^{29,30}$ The peak 1 at $\delta 8.642$ with singlet(s) line shape is possibly a $\mathrm{CH}\left(N_{1}=1\right)$ and an ideal candidate to start the search. When $A_{1}$ (0.696) was used as a divisor (Eq. 3), three peaks, 4( $\delta 7.376$, d-doublet, $\left.A_{4}=0.69\right), 10(\delta 4.022$, t-triplet, $\left.A_{10}=0.679\right)$ and $16\left(\delta 3.334\right.$, m-multiplet, $\left.A_{16}=1.35\right)$, had $N_{4,10,16}$ $=1,1,2$ and $\Delta \leq 0.1$, respectively, and could be classified in one group according to the 1 st $\& 2$ nd criteria. The other peaks had either impractical proton number or $\Delta>0.1$. This component corresponds to histidine. Once the group is defined, the average value of peak area to spin number is calculated and the value is directly correlated to the concentration (Eq. 1) of the component. In the next step when peak 2 ( $\delta 7.718, \mathrm{~d}, N_{2}=1$, $\left.A_{2}=0.388\right)$ was used as a divisor, the values of $N$ for seven peaks at $\delta 7.525\left(\mathbf{3}, \mathrm{d}, A_{3}=0.386\right), \delta 7.307\left(\mathbf{5}, \mathrm{s}, A_{5}=0.387\right), \delta 7.27(\mathbf{6}, \mathrm{t}$, $\left.A_{6}=0.383\right), \delta 7.187$ (7, t, $\left.A_{7}=0.393\right), \delta 4.066\left(\mathbf{9}, \mathrm{dd}, A_{9}=0.395\right)$ $\delta 3.475\left(\mathbf{1 5}, \mathrm{dd}, A_{15}=0.376\right)$ and $\delta 3.301\left(\mathbf{1 7}, \mathrm{dd}, A_{17}=0.358\right)$ were found to be $1: 1: 1: 1: 1: 1: 1(\mathrm{CH})$. This component is assigned to be tryptophan. Next we took peak 22 at $\delta 1.673(\mathrm{~m})$, as an example for starting the search with a methylene group. This assumption is reasonable because of its symmetrical line shape in profile but different $J_{\mathrm{s}}$ from two halves can be inferred to arise from two magnetically inequivalent protons strongly coupled with each other but weakly coupled with other protons. When this peak $\left(N_{22}=2, A_{22}=1.06\right)$ was applied, we found that the values of $N$ for three peaks at $\delta 3.767\left(\mathbf{1 2}, \mathrm{t}, A_{12}=0.538\right), \delta 3.232(\mathbf{1 8}$, q, $\left.A_{18}=1.05\right)$ and $\delta 1.898\left(\mathbf{2 1}, \mathrm{m}, A_{21}=1.06\right)$ were found to be 1 , 2 and 2 . These peaks were assigned to arginine. Similarly, three subgroups of peaks, group $1\left(\mathbf{1 1}, A_{11}=3.87, \mathbf{1 9}, A_{19}=7.30, \mathbf{2 0}\right.$, $\left.A_{20}=7.29\right)$, group $2\left(\mathbf{1 4}, A_{14}=0.842\right)$ and group $3\left(\mathbf{8}, A_{8}=0.346\right.$, 13, $\left.A_{13}=0.346,23, A_{23}=0.998\right)$ can be found which correspond 
to L-threonine, glycine and L-glutamate, respectively. It should be indicated that the divisor may be varied within defined error to see whether or not more peaks belong to one component. That is exactly what our customized local optimization algorithm does. But if one peak satisfies both criteria belongs to two or more components, our customized algorithm for global optimization will select and recommend the solution with minimal error. After the $N$ was defined for each peak, relative concentrations were derived directly for all components using Eq. 1a (Table S1), which in turn were used to construct CORDY. Then absolute concentration of other components can be determined from only one component of known concentration, here for example L-threonine $(4.85 \mathrm{mM})$. It can be seen from Figure 1 that CORDY provides a separation capacity of at least one order of magnitude in the $C$ dimension, resulting in a significant partition of six components of the sample.

To further demonstrate the usefulness of CORDY, an energy drink namely sugar-free Red $B u l l^{\circledR}$ was taken as another example. After careful phase and baseline correction of a fully relaxed ${ }^{1} \mathrm{H}$ NMR spectrum (Figure 2a), twenty-two peaks were integrated (Table S1) where the overlapped peaks $11 \& 12$ were resolved by deconvolution as mentioned before. The peak $\mathbf{1}$ at $\delta 8.943$ with singlet(s) line shape is possibly a $\mathrm{CH}\left(N_{1}=1\right)$ and an ideal candidate to start the search. When $A_{1}(2.46)$ was used as divisor (Eq. 3), three peaks; 2 ( $\delta 8.703$, doublet, d), 3 ( $\delta 8.405$, d) and $\mathbf{5}$ ( $\delta 8.716$, triplet, t), had $N_{2,3,5}=1$ and $\Delta \leq 0.1$, respectively and could be classified in one group according to the $1^{\text {st }}$ $\& 2^{\text {nd }}$ criteria. The other peaks had either impractical proton number or $\Delta>0.1$. Referring to ingredients of Red Bull ${ }^{\circledR}$, chemical shifts, line-shapes of these peaks and literature, ${ }^{31}$ the peaks $1,2,3$ \& 5 were assigned to $2-\mathrm{H}, 6-\mathrm{H}, 4-\mathrm{H} \& 5-\mathrm{H}$ of niacin (vitamin B3), respectively. Once the group is defined, the average value of peak area divided by spin number can be calculated and is directly correlated to concentration (Eq. 1) of the component. The mean value of niacin was set to 1.0 for internal reference. Using peak $4\left(\delta 7.804, \mathrm{~s}, N_{4}=1, A_{4}=15.0\right)$ as a divisor, the value of $N$ for three peaks at $\delta 3.861(7, \mathrm{~s}), \delta 3.441(\mathbf{1 2}, \mathrm{s})$ and $83.259(\mathbf{1 4}, \mathrm{s})$ was found to be $3\left(\mathrm{CH}_{3}\right)$. These four peaks were originated from caffeine $\mathrm{e}^{32,33}\left(8-\mathrm{CH}, 7-\mathrm{CH}_{3}, 1-\mathrm{CH}_{3}\right.$ \& $3-$ $\mathrm{CH}_{3}$, respectively), an indispensable component of $\mathrm{Red} \mathrm{Bull}{ }^{\circledR}$ with relative concentration of 6.02. We took peak 22 at $\delta 1.044$ (d), as an example for starting the search with a methyl group. When this peak $\left(N_{22}=3, A_{22}=540.3\right)$ was applied, it was found that the peaks $8\left(\delta 3.786, \mathrm{~m}, N_{8}=1\right), \mathbf{1 1}\left(\delta 3.448, \mathrm{dd}, N_{11}=1\right)$ and 13 ( $\delta 3.348$, dd, $\left.N_{13}=1\right)$, belonged to the same group of 22 with relative concentration of 73.7. The peak $\mathbf{8}$ was assigned to 2$\mathrm{CH}, \mathbf{1 1}$ and 13 to two geminate protons $1-\mathrm{CH}_{2}$, and 22 to $3-\mathrm{CH}_{3}$, respectively of propylene glycol. ${ }^{34,35}$ Using the same procedure, we were able to separate the leftover assigned peaks into four groups, defined the $N$, relative concentration and assignd them $^{31,36,37}$ to acesulfame-K $\left(N_{6}=1, N_{18}=3\right)$, arginine $\left(N_{9}=1\right.$, $\left.N_{15}=2, N_{19}=2, N_{20}=2\right)$, citrate $\left(\mathrm{N}_{16}=2, \mathrm{~N}_{17}=2\right)$ and ethanol $\left(N_{10}=1\right.$, $\left.N_{21}=3\right)$, respectively. The typical chemical shifts $(\delta 2.810$, $\delta 2.687)$ and coupling constant $(J=15.5 \mathrm{~Hz})$ make it easy to assign the peaks to two geminate protons $1,3-\mathrm{CH}_{2}$ of citrate. ${ }^{31}$ After the $N$ was defined for each peak, relative concentrations, referring to niacin as 1.00 , were derived directly for all components using Eq. 1a (Table S1), which in turn were used to construct CORDY. It can be seen from Figure 2 that CORDY provides a separation capacity of at least two orders of magnitude in the $C$ dimension, resulting in a good separation of seven components of the sample.
For comparison, DOSY was conducted and shown in Figure S2 which separated the seven components into four groups, with ethanol alone in a group, niacin, acesulfame-K and propylene glycol in the second group, caffeine in the third group, citrate and arginine in the fourth group, possibly due to the small dynamic range of the $D$ values $(6.3 \sim 10 \times 10 \quad 10 \mathrm{~m} 2 / \mathrm{s})$. In general, components with similar self-diffusion coefficients or concentration could not be resolved by DOSY or CORDY, respectively.

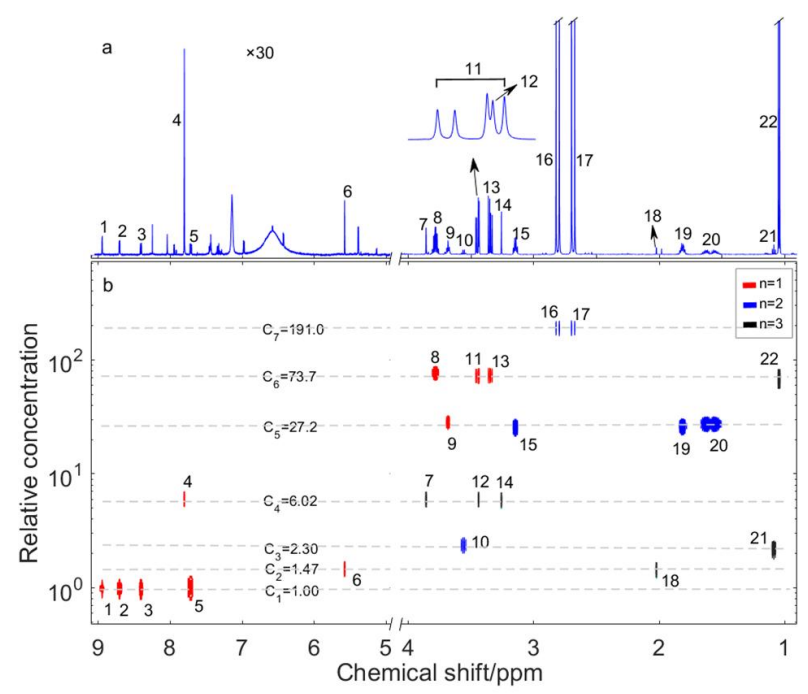

Figure 2. High resolution fully relaxed $1 \mathrm{H}$ NMR spectrum (a) of sugar-free Red Bull ${ }^{\circledR}$ and its CORDY (b). The vertical scale was magnified by 30 times on the left side. The overlapped peaks 11 \& 12 are enlarged and plotted as inserts. The peaks are separated into seven groups according to relative concentrations and assigned to niacin $(1,2,3,5 ; \mathrm{C}=1.0$ as reference), caffeine $(4,7,12,14)$, acesulfame-K $(6,18)$, arginine $(9,15,19,20)$, ethanol $(10,21)$, citrate $(16,17)$, propylene glycol $(8,11,13,22)$, respectively.

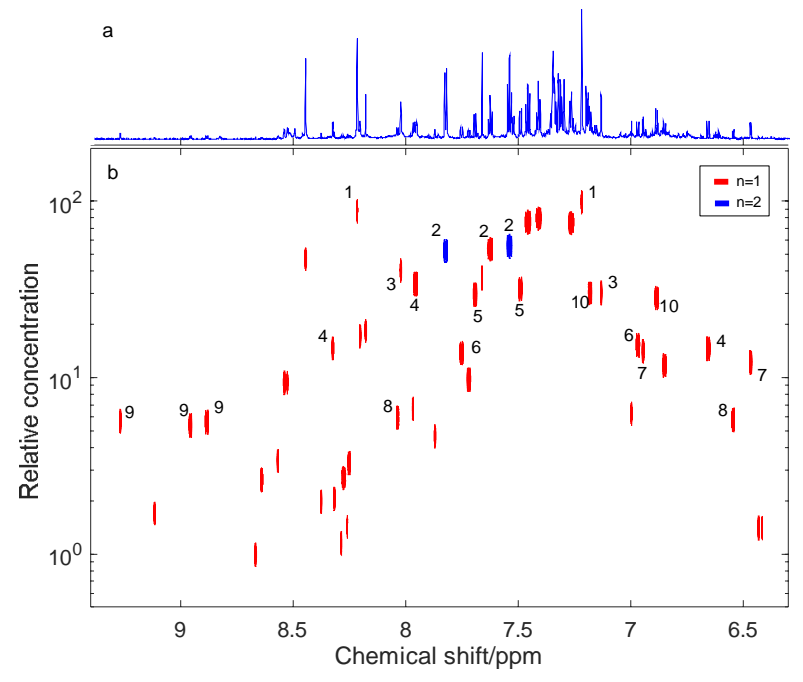

Figure 3. ${ }^{1} \mathrm{H}$ CORDY plot (b) of human urine spectrum (a) in the region (6.3 9.4 ppm). Ten molecules in TOCSY are labelled by numbers. The molecules 2 and 3 are hippurate and $\pi$-methylhistidine, respectively. For the assignment of other peaks see Table $\mathrm{S} 1$. 
For more complex mixtures, human urine was taken as an example and the less overlapped region (6.3 9.4ppm) was selected for analysis. The analysis is greatly simplified because only $\mathrm{CH}$ groups exist. We employed the commercial software MNOVA to automatically deconvolute the spectrum and picked 47 multiplets regardless of mild peak overlaps. Two strongly coupled multiplets (hippurate) were easily identified and their number of protons was set as two. Regardless of magnetically equivalent protons, for simplicity the integral value of multiplet is directly used as the vertical coordinate for plotting CORDY (Figure 3) with linewidth in indirect dimension set as $7 \%$ of the integral value. The peaks belonging to the same coupling system in TOCSY have been labelled in Figure 3. The three peaks of component 4 are $\mathrm{CH}, 2 \mathrm{CH}$ and $\mathrm{CH}$, respectively. The results show that our proposed method is equally useful for the resolution of complex biological mixtures.

The results stated above, prove that CORDY is especially useful for mixtures of high dynamic range. Two components with similar concentration may fail to be resolved but could be separated by other dimension, such as diffusion coefficient. In practice, it is less likely to have two components with both similar concentration and diffusion coefficient. This results in a possibility of enhancing resolution of both CORDY and DOSY by the combination of two approaches, resulting in three dimensional concentration and diffusion ordered spectroscopy CORDY-DOSY. CORDY-DOSY is obtained by post-processing with combination of CORDY and DOSY. This provides flexible options for processing and displaying full range or part of the concentration and the self-diffusion coefficient, with high resolution in the chemical shift dimension. For clarity and simplicity, DOSY only shows $D$ value of the peak against its integral range. Figure $4 \mathrm{a}$ and $4 \mathrm{~b}$ show the $1 \mathrm{D}$ fully relaxed ${ }^{1} \mathrm{H} N \mathrm{NMR}$ spectrum and the DOSY of the amino acids mixture. The peaks of glycine (14) and threonine $(\mathbf{8}, \mathbf{1 3} \& \mathbf{2 3})$ were resolved and appeared on the top of DOSY (Fig. 4b). Due to the similar $D$ values, the peaks of histidine $(\mathbf{1}, \mathbf{4}, \mathbf{1 0} \& \mathbf{1 6})$ and glutamate $(\mathbf{1 1}$, $19 \& 20)$, tryptophan $(2,3,5,6,7,9,15 \& 17)$ and arginine $(12$, 18, $21 \& 22$ ) are overlapped in diffusion dimension and labelled with dashed rectangles in Fig. 4b. However, the overlapped regions were fully resolved in CORDY (Fig. $4 \mathrm{c} \& 4 \mathrm{~d}$ ). On the other side, the concentrations of threonine $(4.85 \mathrm{mM})$ and tryptophan $(5.44 \mathrm{mM})$ were close and hardly distinguishable in CORDY (Fig. 1), but were unambiguously separated in DOSY (Fig. 4b). Therefore, the enhanced resolution by combination of both CORDY and DOSY is validated by the model mixture of six amino acids as well as the sugar-free $\operatorname{Red} B u l^{(\mathrm{R})}$ sample.

In a summary, a novel approach, CORDY, was proposed based on the principle that ratio of the peak area to its associated spin number is proportional to the concentration of the molecule, a unique property of NMR spectroscopy. The new method was validated on a model mixture consisted of six amino acids, human urine and a sugar-free energy drink Red Bull ${ }^{\circledR}$. It was demonstrated that CORDY provides a new way for simultaneous quantitation of the components and separation of their NMR peaks in a mixture, resulting in high dynamic range of more than one or two orders of magnitude in the concentration dimension and high-resolution in chemical shift dimension. In addition, CORDY could be combined with DOSY to further resolve the components with similar concentration or self-diffusion coefficient. It should be noted that with the help of deconvolution, it is only possible to resolve the partially overlapped peaks.

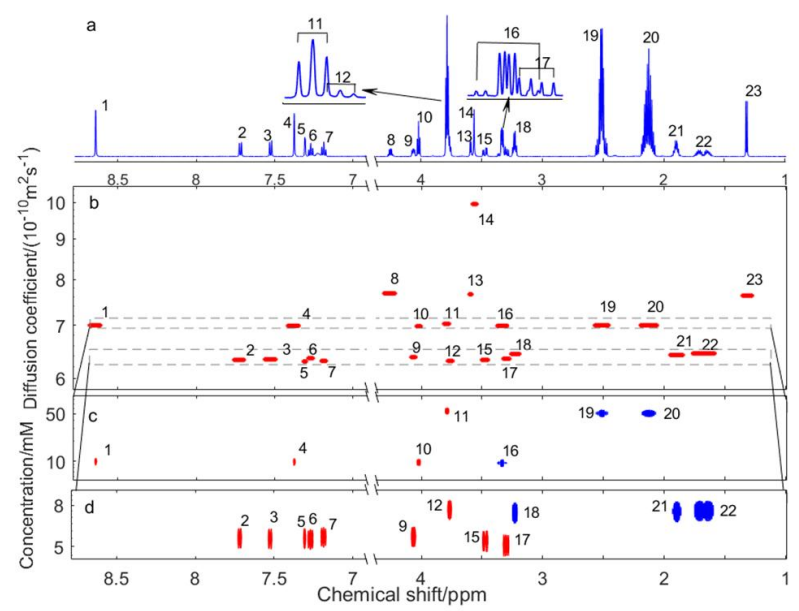

Figure 4. (A) High resolution $1 \mathrm{H}$ NMR spectrum showing the spectral region $(1.0 \sim 8.7 \mathrm{ppm})$ with two enlarged parts after resolution enhancement. (B) Diffusion coefficients of the peaks plotted against integral range. (C) and (D) are CORDY plots of two subregions labelled by dashed rectangle in (B). The peaks are assigned: L-threonine $(\mathbf{8}, \mathbf{1 3}, \mathbf{2 3})$, L-glutamate $(\mathbf{1 1}, \mathbf{1 9}, \mathbf{2 0})$, L-histidine $(\mathbf{1}, \mathbf{4}$, 10, 16), L-tryptophan $(2,3,5,6,7,9,15,17)$, L-arginine $(12,18$, $21,22)$ and glycine $(\mathbf{1 4})$. The concentration value of components is referenced to L-threonine $(4.85 \mathrm{mM})$

\section{EXPERIMENTAL SECTION}

\section{Sample Preparation}

Amino acids, L-Histidine, L-Threonine, L-Arginine, L-Glycine, L-Tryptophan and L-Glutamic, and D2O (99.9\%) were purchased from Sigma (St. Louis, MO) and Biosharp (Hefei, China). Double distilled water was prepared on a Millipore system and was used as solvent for amino acids. Energy drink sugar-free Red Bull@ was manufactured in Switzerland according to the information printed on label of the can and purchased from Jingdong Worldwide (Jingdong's import cross-border ecommerce platform). Human urine was provided by the first author.

The first sample consisted of $250 \mu 1 \mathrm{D}_{2} \mathrm{O}, 300 \mu 1$ human urine and sodium azide of $0.05 \% \mathrm{w} / \mathrm{v}$. The second testing sample was consisted of $100 \mu 1 \mathrm{D}_{2} \mathrm{O}$ and $400 \mu 1$ Red Bull@. The human urine sample was prepared after filtering using 0.22 um microfiltration membrane. The sample of amino acid mixture was prepared in aqueous solution with addition of $17.8 \%(\mathrm{v} / \mathrm{v}) \mathrm{D}_{2} \mathrm{O}$ for field lock. The concentration of threonine was $4.85 \mathrm{mM}$ and used as internal reference.

\section{NMR Experiments}

All NMR experiments were performed on Bruker Avance HQ spectrometers (Bruker Biospin, Germany) operating, respectively, at ${ }^{1} \mathrm{H}$ frequency of $599.81 \mathrm{MHz}$ (Oxford Magnet for the amino acid mixture), $949.9 \mathrm{MHz}$ (for human urine) and $600.13 \mathrm{MHz}$ (for the Red Bullß) at $25^{\circ} \mathrm{C}$, all equipped with CryoProbe. High resolution 1D NMR spectra and the ones for self-diffusion coefficient measurement (DOSY) were acquired by using the pulse sequences of the ZGPR/modified ZGGPPR and BPP-LED, respectively. The detailed parameters for data acquisition and processing have been provided in supporting information. 


\section{ACKNOWLEDGEMENTS}

This research was funded by National Natural Science Foundation of China (21735007, 21991080, 21921004), National Key R\&D Program of China (2018YFE02202300, 2018YFA0704002, 2017YFA0505400), and CAS Key Research Program of Frontier Sciences (QYZDJ-SSW-SLH027).

All NMR experiments on the $950 \mathrm{MHz}$ spectrometer were performed at the NMR facility of the National Center for Protein Sciences at Peking University.

\section{Supporting Information}

The Supporting Information is available free of charge on the ACS Publications website.

\section{Key words}

NMR, mixture, quantitative, separation, concentration

\section{REFERENCES}

(1) Abragam, A. The principles of nuclear magnetism; Oxford University Press: London, 1961.

(2) Ersnt, R. R.; Bodenhausen, G.; Wokaun, A. Principles of Nuclear Magnetic Resonance in One and Two Dimensions; Clarendon Press, Oxford University Press: New York and London, 1987, p 610.

(3) Callaghan, P. T. Principles of Nuclear Magnetic Resonance Microscopy; Claredon Press, Oxford, 1993.

(4) Simmler, C.; Napolitano, J. G.; McAlpine, J. B.; Chen, S. N.; Pauli, G. F. Curr. Opin. Biotechnol. 2014, 25, 51-59.

(5) Li, X.; Hu, K. In Annual Reports on Nmr Spectroscopy, Vol 90, Webb, G. A., Ed., 2017, pp 85-143.

(6) Mulder, F. A. A.; Tenori, L.; Luchinat, C. Angew. Chem. Int. Ed. 2019, 58, 15283-15286.

(7) Aue, W. P.; Bartholdi, E.; Ernst, R. R. J. Chem. Phys. 1976, 64, 2229-2246.

(8) Nicholson, J. K.; Wilson, I. D. Prog. Nucl. Magn. Reson. Spectrosc. 1989, 21, 449-501.

(9) Fan, W. M. T. Prog. Nucl. Magn. Reson. Spectrosc. 1996, 28, 161-219.

(10) Braunschweiler, L.; Ernst, R. R. Journal of magnetic resonance 1983, 53, 521-528.

(11) Jeener, J.; Meier, B. H.; Bachmann, P.; Ernst, R. R. J. Chem. Phys. 1979, 71, 4546-4553.

(12) Stejskal, E. O.; Tanner, J. E. J. Chem. Phys. 1965, 42, 288292.

(13) Morris, K. F.; Johnson, C. S. J. Am. Chem. Soc. 1992, 114, 3139-3141.

(14) Barjat, H.; Morris, G. A.; Swanson, A. G. J. Magn. Reson. 1998, 131, 131-138.

(15) Loening, N. M.; Keeler, J.; Morris, G. A. Journal of magnetic resonance 2001, 153, 103-112.

(16) Peresada, S.; Tonielli, A.; Morici, R.; Johnson, C. S. Progress in nuclear magnetic resonance spectroscopy 1999, 34, 203-256.

(17) Liu, M. L.; Nicholson, J. K.; London, J. C. Analytical Chemistry 1996, 68, 3370-3376.

(18) Zhang, X.; Li, C. G.; Ye, C. H.; Liu, M. L. Analytical Chemistry 2001, 73, 3528-3534.

(19) Nilsson, M.; Botana, A.; Morris, G. A. Analytical Chemistry 2009, 81, 8119-8125.

(20) Cloarec, O.; Dumas, M. E.; Craig, A.; Barton, R. H.; Trygg, J.; Hudson, J.; Blancher, C.; Gauguier, D.; Lindon, J. C.; Holmes, E.; Nicholson, J. Anal. Chem. 2005, 77, 1282-1289.

(21) Maher, A. D.; Fonville, J. M.; Coen, M.; Lindon, J. C.; Rae, C. D.; Nicholson, J. K. Analytical Chemistry 2012, 84, 10831091.
(22) Robinette, S. L.; Lindon, J. C.; Nicholson, J. K. Anal. Chem. 2013, 85, 5297-5303.

(23) Holmes, E.; Cloarec, O.; Nicholson, J. K. J. Proteome Res. 2006, 5, 1313-1320.

(24) He, Q. H.; Johnson, C. S. J. Magn. Reson. 1989, 81, 435439.

(25) Morris, K. F.; Johnson, C. S. J. Magn. Reson., Ser A 1993 , 101, 67-73.

(26) Heil, S. R.; Holz, M. Angew. Chem.-Int. Edit. Engl. 1996, 35, $1717-1720$

(27) Fang, Y.; Yushmanov, P. V.; Furo, I. Magnetic Resonance in Chemistry 2017, 55, 584-588.

(28) Badertscher, M.; Bühlmann, P.; Pretsch, E. Structure Determination of Organic Compounds: Tables of Spectral Data, 4th ed.; Springer, Berlin, Heidelberg, 2009.

(29) Morris, G. A.; Barjat, H.; Horne, T. J. Prog. Nucl. Magn. Reson. Spectrosc. 1997, 31, 197-257.

(30) Liu, M. L.; Tang, H. R.; Nicholson, J. K.; Lindon, J. C. Magn. Reson. Chem. 2002, 40, S83-S88.

(31) Simpson, A. J.; Shirzadi, A.; Burrow, T. E.; Dicks, A. P.; Lefebvre, B.; Corrin, T. J. Chem. Educ. 2009, 86, 360-362.

(32) Kan, L. S.; Borer, P. N.; Cheng, D. M.; Tso, P. O. P. Biopolymers 1980, 19, 1641-1654.

(33) D'Amelio, N.; Fontanive, L.; Uggeri, F.; Suggi-Liverani, F.; Navarini, L. Food Biophys. 2009, 4, 321-330.

(34) Dauner, B. R.; Pringle, D. L. J. Chem. Educ. 2014, 91, 743746.

(35) Zhou, Y.; Hu, K.; Shen, J. F.; Wu, X. J.; Cheng, G. Z. J. Mol. Struct. 2009, 921, 150-155.

(36) Ohtsuki, T.; Sato, K.; Abe, Y.; Sugimoto, N.; Akiyama, H. Talanta 2015, 131, 712-718.

(37) Liu, M. L.; Nicholson, J. K.; Parkinson, J. A.; Lindon, J. C. Anal. Chem. 1997, 69, 1504-1509. 